\title{
Modeling of X-ray excited luminescence intensity dependence on the nanoparticle size
}

Article in Radiation Measurements · December 2015

DOI: 10.1016/j.radmeas.2015.12.010

CITATIONS

0

10 authors, including:

\section{Stepan Syrotyuk}

Lviv Polytechnic

41 PUBLICATIONS 41 CITATIONS

SEE PROFILE
READS

51

\section{A. Gektin}

National Academy of Sciences of Ukraine

121 PUBLICATIONS 600 CITATIONS

SEE PROFILE

All content following this page was uploaded by Stepan Syrotyuk on 21 December 2015. 


\section{Accepted Manuscript}

Modeling of X-ray excited luminescence intensity dependence on the nanoparticle size

V. Vistovskyy, Ya. Chornodolskyy, A. Gloskovskii, S. Syrotyuk, T. Malyi, M. Chylii, P. Zhmurin, A. Gektin, A. Vasil'ev, A. Voloshinovskii

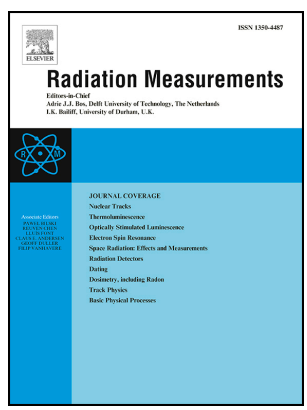

PII:

S1350-4487(15)30089-5

DOI:

10.1016/j.radmeas.2015.12.010

Reference: $\quad$ RM 5491

To appear in: Radiation Measurements

Received Date: 12 October 2015

Revised Date: 8 December 2015

Accepted Date: 15 December 2015

Please cite this article as: Vistovskyy, V., Chornodolskyy, Y., Gloskovskii, A., Syrotyuk, S., Malyi, T., Chylii, M., Zhmurin, P., Gektin, A., Vasil'ev, A., Voloshinovskii, A., Modeling of X-ray excited luminescence intensity dependence on the nanoparticle size, Radiation Measurements (2016), doi: 10.1016/j.radmeas.2015.12.010.

This is a PDF file of an unedited manuscript that has been accepted for publication. As a service to our customers we are providing this early version of the manuscript. The manuscript will undergo copyediting, typesetting, and review of the resulting proof before it is published in its final form. Please note that during the production process errors may be discovered which could affect the content, and all legal disclaimers that apply to the journal pertain. 


\title{
Modeling of X-ray excited luminescence intensity dependence on the
}

\section{nanoparticle size}

V. Vistovskyy ${ }^{\mathrm{a}, 1}$, Ya. Chornodolskyy ${ }^{\mathrm{a}}$, A. Gloskovskii ${ }^{\mathrm{b}}$, S. Syrotyuk ${ }^{\mathrm{c}}$, T. Malyi ${ }^{\mathrm{a}}$, M. Chylii ${ }^{\mathrm{a}}$, P. Zhmurin ${ }^{\mathrm{d}}$, A. Gektin ${ }^{\mathrm{d}}$, A. Vasil'ev $^{\mathrm{e}}$, A. Voloshinovskii ${ }^{\mathrm{a}}$

${ }^{a}$ Ivan Franko National University of Lviv, Lviv, Ukraine

${ }^{b}$ Deutsches Elektronen-Synchrotron DESY, 22607 Hamburg, Germany

${ }^{c}$ Lviv Polytechnic National University, Lviv, Ukraine

${ }^{\mathrm{d}}$ Institute for Scintillation Materials, Kharkiv, Ukraine

${ }^{\mathrm{e}}$ Institute of Nuclear Physics, Moscow State University, Moscow, Russia

\begin{abstract}
The thermalization length distribution of electrons over their kinetic energy in a conduction band is calculated on the basis of the data on the electron effective mass, density of states in conduction band, dielectric permittivity and energy of longitudinal optical phonons. The method of modeling of a recombinational luminescence intensity dependence on the nanoparticle size is proposed on the basis of the assumption that the contribution to a recombinational luminescence gives only those charge carriers which in the result of thermalization did not reach a near-surface layer of nanoparticles. Using such the approach the theoretical dependence of recombinational luminescence intensity on the nanoparticle size for $\mathrm{LaPO}_{4}$ and $\mathrm{LuPO}_{4}$ are calculated. The revealed correlation of experimental and theoretical dependences confirms that the commensurability of electron thermalization length with nanoparticle size is the main reason of the sharp decrease of X-ray excited luminescence intensity when the nanoparticle size decreases.
\end{abstract}

Keywords: $\mathrm{LaPO}_{4}, \mathrm{LuPO}_{4}$, nanoparticles, X-ray excited luminescence, energy band structure, electron thermalization length

\section{Introduction}

Studies of the nanoparticle luminescence parameters dependence on their size are driven by a possibility to use the nanoparticles in particular as luminescent biolabels, nanoscintillators for radiotheraphy or components of the composite nanostructured scintillators. For an impurity luminescence in case of intracenter excitation it is known that the intensity of the emission usually decreases with the reduction of the nanoparticle sizes. It is explained with a nonradiative relaxation of the luminescent centers due to the interaction with surface defects. However the drop

\footnotetext{
${ }^{1}$ Corresponding author. Tel.:+380 322964366; fax.:+380 322978903; e-mail: vistvv@ gmail.com
} 
in luminescence intensity when the nanoparticle size decreases in case of intracenter excitation is not as notable as in a case of band-to-band or X-ray excitation. If excitation energy is transferred to the luminescence centers by free charge carriers, the new channel of a nonradiative relaxation appears: created free charge carriers can reach a nanoparticle surface, where they have an opportunity to transfer excitation energy to surface defects or to leave a nanoparticle. It results in strong dependence of a recombinational luminescence on the nanoparticle size. The probability to reach a surface by free charge carriers is determined by the ratio of thermalization length (distance passed by an electron or a hole in the thermalization process) to the nanoparticle size. The thermalization length of holes is considered to be rather small (for alkali halides it is estimated as a few nanometers [1]) due to high effective masses and possible self-trapping processes. Therefore, the thermalization length of electrons is considered as a decisive factor for the scintillation process in nanoparticles.

For verification of this assumption the attempt to simulate the dependences of $\mathrm{X}$-ray excited luminescence intensity on the nanoparticle size for $\mathrm{LaPO}_{4}-\mathrm{Pr}$ and $\mathrm{LuPO}_{4}-\mathrm{Pr}$ which are considered as possible candidates for use as nanoscintillators for radiotheraphy is made in this work. For such possible practical use the dependence of X-ray excited luminescence intensity on their size is studied (Fig. 1). Nanoparticles were obtained and characterized as described in $[2,3]$. As one can see from the figure the intensity of X-ray excited luminescence sharply decreases in the range of 15-40 $\mathrm{nm}$ nanoparticle sizes. According to the assumption these values of the nanoparticle sizes should be commensurate to the average electron thermalization length.

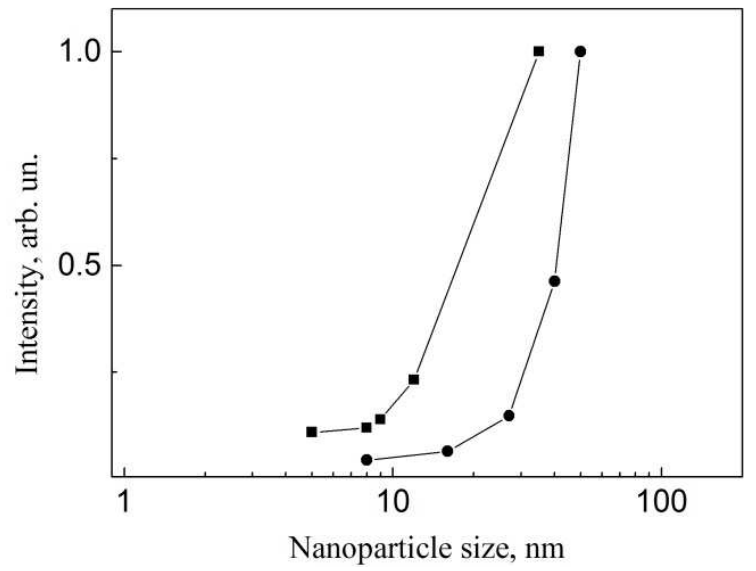


Fig. 1. Dependence of X-ray excited luminescence intensity on the nanoparticle size for $\mathrm{LaPO}_{4}-\mathrm{Pr}$ (circles) and $\mathrm{LuPO}_{4}-\operatorname{Pr}$ (squares).

\section{Calculations of thermalization lengths distribution for secondary} electrons

The thermalization lengths distribution of secondary electrons was calculated for simulation of X-ray excited luminescence intensity dependence on the size of nanoparticles. Nowadays the theory necessary for an assessment of electron thermalization length is quite well developed. For calculation of average thermalization length of electrons with initial kinetic energy $E_{e 0}$ for the case of one branch of longitudinal optical phonons the following equation is used:

$$
l_{e, L O}^{2}\left(E_{e 0}\right)=\frac{8}{9} a_{B}^{2}\left(\frac{\tilde{\varepsilon}}{m_{e}^{*} / m_{0}}\right)^{2} \tanh \left(\frac{\hbar \Omega_{L O}}{2 k_{B} T}\right) \cdot\left(\frac{E_{e 0}}{\hbar \Omega_{L O}}\right)^{3} / \ln \left(\frac{4 E_{e 0}}{\hbar \Omega_{L O}}\right),
$$

where $a_{B}-$ Bohr radius, $\widetilde{\varepsilon}-$ effective dielectric permeability $\left(\widetilde{\varepsilon}^{-1} \equiv \varepsilon_{\infty}^{-1}-\varepsilon_{s t}^{-1}, \varepsilon_{\infty}-\right.$ optical dielectric permeability, $\varepsilon_{s t}$ - static dielectric permeability), $m_{e}^{*}-$ effective mass of an electron, $m_{0}$ - mass of an electron, $\hbar \Omega_{L O}$ - energy of optical phonons [4].

In our estimations we take into account only one phonon branch possessing the highest energy. The thermalization on phonons with lower energies was not taken into account. In result of such approximation the obtained values of thermalization length will be slightly underestimated (see Fig. 6 in [4]).

The input data for calculation of electron thermalization length distribution such as density of electronic states in conduction band and the effective mass of an electron are obtained from electronic energy structure of $\mathrm{LnPO}_{4}(\mathrm{Ln}=\mathrm{La}, \mathrm{Lu})$ crystals. For a $\mathrm{LaPO}_{4}$ crystal the electronic energy structure from [5] was used. The electronic energy spectrum for a $\mathrm{LuPO}_{4}$ crystal (Fig. 2) was calculated, using the PAW formalism [6] taking into account the strong local correlations in a PBE0 GGA approach. The effective mass of electrons for $\mathrm{LnPO}_{4}$ crystals was estimated from dispersion of the lower branch of conduction band at $\Gamma$-point. The effective masses and other parameters of the crystals are specified in Table 1. 


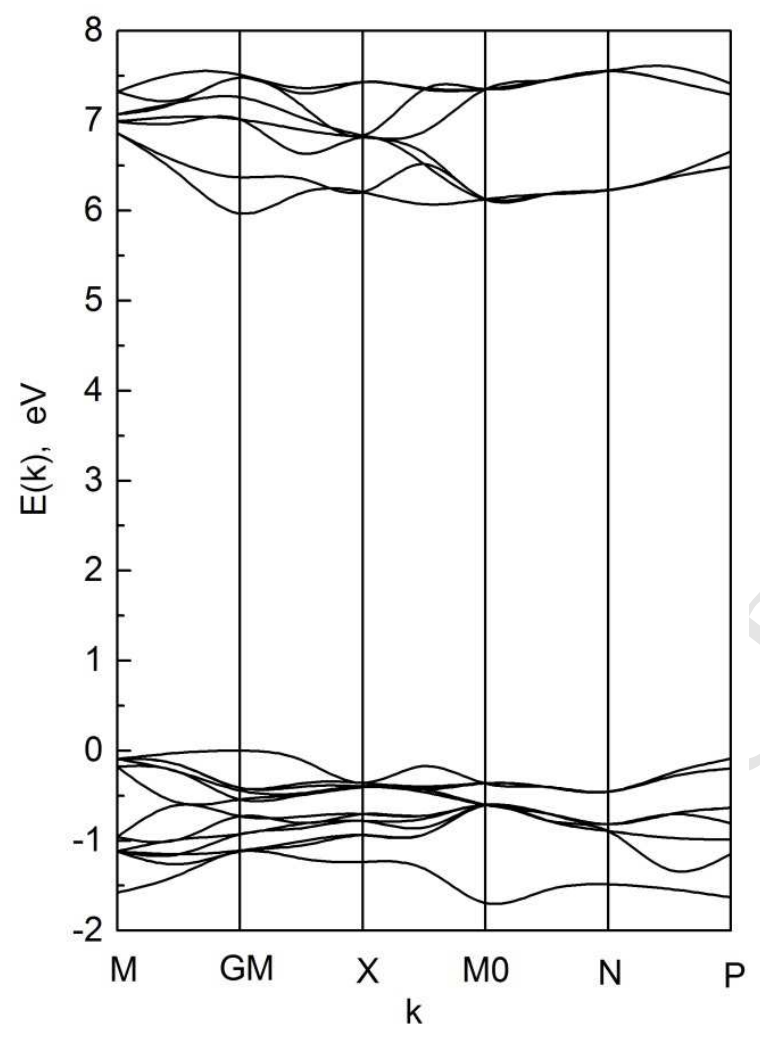

Fig. 2. Energy band structure of a $\mathrm{LuPO}_{4}$ crystal

Table 1. Optical and scintillation parameters of $\mathrm{LnPO}_{4}\left(\mathrm{Ln}=\mathrm{La}\right.$, Lu) crystals: $\mathrm{m} * / \mathrm{m}_{0}-$ ratio of effective mass to the mass of an electron, $n-$ index of refraction, $\varepsilon_{\infty}=n^{2}-$ optical dielectric permeability, $\varepsilon_{\mathrm{st}}$ - static dielectric permeability, $\tilde{\varepsilon}-$ effective dielectric permeability $\left(\widetilde{\varepsilon}^{-1} \equiv \varepsilon_{\infty}^{-1}-\varepsilon_{s t}^{-1}\right), \hbar \Omega_{L O}-$ energy of longitudinal optical phonons.

\begin{tabular}{|l|l|l|c|c|c|c|c|}
\hline & $\mathrm{E}_{\mathrm{g}}, \mathrm{eV}$ & $\mathrm{m} / \mathrm{m}_{0}$ & $\mathrm{n}$ & $\varepsilon_{\infty}$ & $\varepsilon_{\mathrm{st}}$ & $\tilde{\varepsilon}$ & $\hbar \Omega_{\mathrm{LO}}, \mathrm{eV}$ \\
\hline $\mathrm{LaPO}_{4}$ & 8,4 & 3,3801 & $1,79[7]$ & 3,204 & $11,1[8]$ & 4,504 & $0,1289[9]$ \\
\hline $\mathrm{LuPO}_{4}$ & 8,8 & 3,4559 & $1,68[10]$ & 2,822 & $10,58[11]$ & 3,848 & $0,1436[10]$ \\
\hline
\end{tabular}

Calculations of thermalization length distribution for secondary electrons were carried out by the technique described in [4] with only one difference: the density of electronic states in conduction band was taken into account for distribution of secondary electrons over kinetic energy. Instead of the $n\left(E_{e 0}\right)$ function [4] the $\mathrm{n}\left(\mathrm{E}_{\mathrm{e} 0}\right) \cdot \mathrm{g}(\mathrm{E})$ product, where $\mathrm{g}(\mathrm{E})$ - density of electronic states in conduction band (Fig. 3), was taken. Results of calculation of secondary electrons distribution over thermalization lengths are presented in Fig. 4. As one can see from the figure the 
average thermalization length of electrons for $\mathrm{LaPO}_{4}$ is smaller in comparison with that for $\mathrm{LuPO}_{4}$.

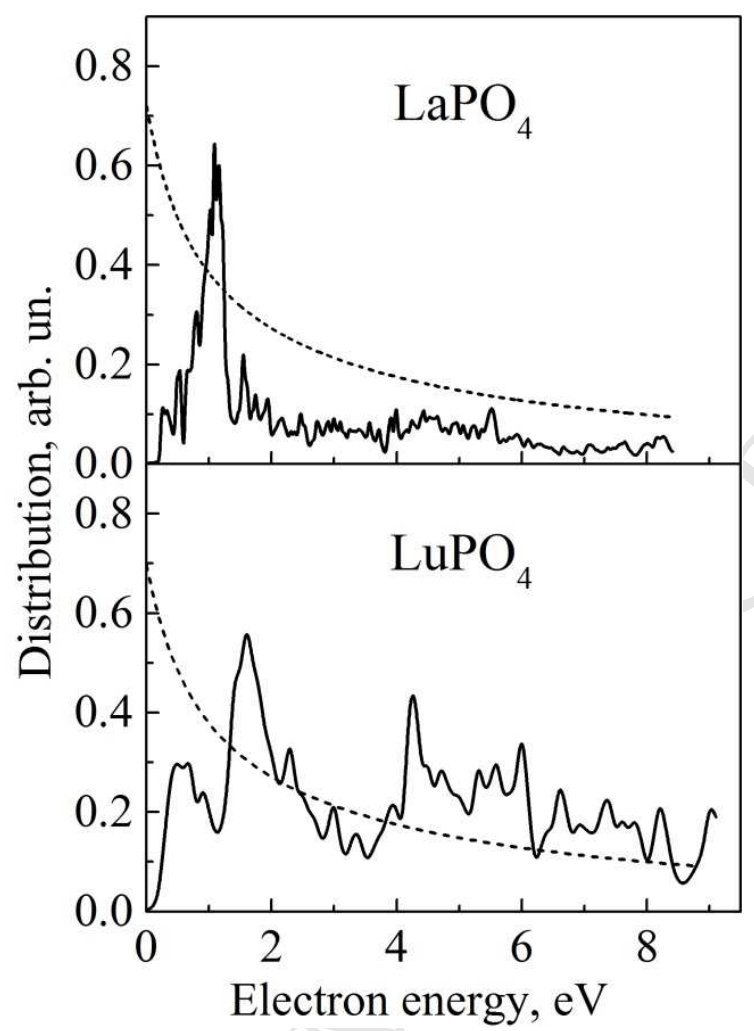

Fig. 3. Density of electronic states in conduction band (solid) and the function of secondary electrons distribution over kinetic energy in the $\left[0, E_{\mathrm{g}}\right]$ range (dash) for $\mathrm{LaPO}_{4}$ and $\mathrm{LuPO}_{4}$ crystals

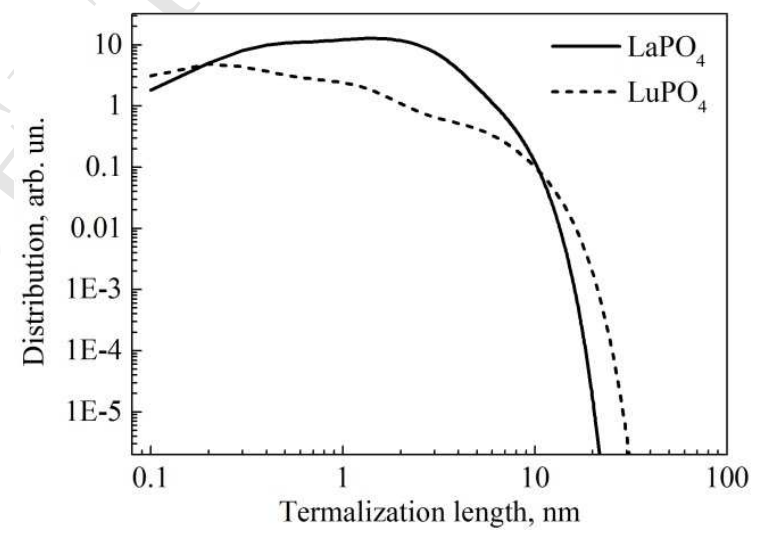

Fig. 4. Distribution of secondary electrons thermalization length for $\mathrm{LnPO}_{4}(\mathrm{Ln}=\mathrm{La}$, $\mathrm{Lu}$ ) crystals

2. Simulation of recombinational luminescence intensity dependence on the nanoparticle sizes 
Dependence of recombinational luminescence intensity on the nanoparticle size

was simulated assuming that the commensurability of secondary electrons thermalization length and the nanoparticle size is the main reason of their luminescence quenching upon the X-ray excitation. In other words, if in the result of thermalization the electron reaches a nanoparticle surface, then its relaxation will be nonradiative, for example, with the participation of surface defects. Also, it is assumed that the formation of excitons or a recombination with impurity centers happens only for thermalized electrons with kinetic energy equal to the energy of optical phonons.

For calculations of X-ray excited luminescence intensity dependence on nanoparticle size the probability $P(R, d)$ of radiative relaxation of a secondary electron formed at distance $d$ from the center of a nanoparticle with the radius $R(R>d)$ was determined in the following way. Let the $l(r)$ function define the thermalization length distribution for secondary electrons of conduction band possessing the kinetic energies in the $[0, E g]$ range, and $l_{m}$ - the maximum value of thermalization length. Let the sphere of the radius $l_{m}$ built with the center in a point of secondary electron creation be called a thermalization sphere. The form of nanoparticles is considered to be spherical. Then the probability $P(R, d)$ of radiative relaxation of a secondary electron will be defined by the intersection area of the thermalization sphere and the sphere of the nanoparticle. In other words, we need to find the part of the thermalization sphere that is within a nanoparticle taking into account "inhomogeneity" of the thermalization sphere, which is defined by the $l(r)$ function. Drawing an analogy between distribution $l(r)$ and the density of a material, the probability of radiative relaxation of a secondary electron $P(R, d)$ was found as the ratio of "mass" of the thermalization sphere part that is within a nanoparticle $m$ to "mass" of all thermalization sphere $m_{0}$.

Based on the above, the probability $P(R, d)$ of the radiative relaxation of a secondary electron formed at the distance $d$ from the center of a nanoparticle with radius $R$ can be obtained using the ratios given below. Integration is carried out in spherical coordinates with origin at the center of the thermalization sphere: 
(i) $d+l_{\mathrm{m}}<R-$ the thermalization sphere is completely within a nanoparticle $=>$ $P(R, d) \equiv 1$

(ii) $R+d<l_{\mathrm{m}}$ - a nanoparticle is completely within the thermalization sphere

$$
P(R, d)=\frac{m}{m_{0}}=\frac{\int_{0}^{\pi} \sin (\theta) d \theta \int_{0}^{d \cos (\theta)+\sqrt{d^{2} \cos ^{2}(\theta)+R^{2}-d^{2}}} l(r) r^{2} d r}{\int_{0}^{\pi} \sin (\theta) d \theta \int_{0}^{l_{m}} l(r) r^{2} d r}
$$

(iii) the thermalization sphere contains only a part of a nanoparticle

$$
\begin{gathered}
P(R, d)=\frac{m}{m_{0}}=\frac{\int_{0}^{\theta_{1}} \sin (\theta) d \theta \int_{0}^{l_{m}} l(r) r^{2} d r}{\int_{0}^{\pi} \sin (\theta) d \theta \int_{0}^{l_{m}} l(r) r^{2} d r}+ \\
+\frac{\int_{\theta_{1}}^{\pi} \sin (\theta) d \theta \int_{0}^{d \cos (\theta)+\sqrt{d^{2} \cos ^{2}(\theta)+R^{2}-d^{2}} l(r) r^{2} d r}}{\int_{0}^{\pi} \sin (\theta) d \theta \int_{0}^{l_{m}} l(r) r^{2} d r}
\end{gathered}
$$

where $\theta_{1}=\arccos \left(\frac{d^{2}+l_{m}{ }^{2}-R^{2}}{2 d l_{m}}\right)$.

Knowing the function $P(R, d)$ the probability of emission of the whole nanoparticle $P(R)$ can be found by integrating $P(R, d)$ over the nanoparticle volume and dividing by its volume. According to the model the function $P(R)$ will describe the dependence of recombinational luminescence intensity on nanoparticle size.

Results of simulation for $\mathrm{LnPO}_{4}(\mathrm{Ln}=\mathrm{La}, \mathrm{Lu})$ nanoparticles are shown in Fig. 5. As one can see, the intensity of a recombinational luminescence starting from a certain nanoparticle size begins to decrease sharply. For the simulated (Fig. 5) and experimental (Fig. 1) data quite good correlation is observed - values of the nanoparticle sizes, in the range of which the sharpest decrease of recombinational luminescence intensity is observed, are $20-50 \mathrm{~nm}$.

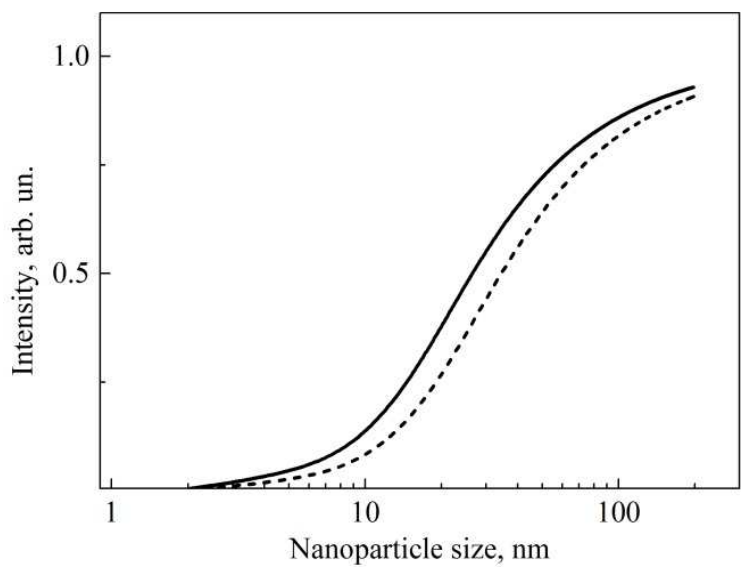


Fig. 5. Calculated dependencies of recombination luminescence intensity on the size of $\mathrm{LaPO}_{4}$ (dash) and $\mathrm{LuPO}_{4}$ (solid) nanoparticles

\section{Conclusions}

The revealed correlation of experimental and simulated dependencies of recombinational luminescence intensity on the nanoparticle size confirms a hypothesis, that the main mechanism of recombinational luminescence quenching is connected with the capture of secondary electrons by the nanoparticle surface defects or their escape from nanoparticles.

From the studied dependencies of recombination luminescence intensities on size of the lanthanide phosphate nanoparticles, it follows that nanoparticles with high energy of optical phonons and effective mass of charge carriers can be perspective nanoscintillators, because the threshold of X-ray luminescence quenching will be observed for such nanoparticles at lower sizes. Considering it, other oxygen containing nanoparticles based on aluminates, silicates, vanadates, and also multicomponent compounds containing several cations can also be perspective materials for nanoscintillators.

\section{References}

[1] G. Bizarri, W. W. Moses, J. Singh, A. N. Vasil'ev, R. T. Williams. An analytical model of nonproportional scintillator light yield in terms of recombination rates. J. Appl. Phys. - 2009. - Vol. 105. - P. 044507.

[2] T. S. Malyy, V. V. Vistovskyy, Z. A. Khapko, A. S. Pushak, N. E. Mitina, A. S. Zaichenko, A. V. Gektin, and A. S. Voloshinovskii. Recombination luminescence of $\mathrm{LaPO}_{4}-\mathrm{Eu}$ and $\mathrm{LaPO}_{4}-\mathrm{Pr}$ nanoparticles. Journal of Applied Physics. - 2013. V.113. - P. 224305.

[3] V. Vistovskyy, T. Malyy, A. Pushak, A. Vas'kiv, A. Shapoval, N. Mitina, A. Gektin, A. Zaichenko, A. Voloshinovskii. Luminescence and scintillation properties of $\mathrm{LuPO}_{4}$-Ce nanoparticles. Journal of Luminescence. - 2014. - Vol. 145. - P. 232-236.

[4] A.N. Vasil'ev, A. Gektin. Multiscale Approach to Estimation of Scintillation Characteristics. IEEE Trans. on Nucl. Sci. - 2014. - Vol. 61. - P. 235-245. 
[5] S.V. Syrotyuk, Y.M. Chornodolskyy, V.V. Vistovskyy, A.S. Voloshinovskii, A.V. Gektin. Band structure of $\mathrm{LaPO}_{4}$. Functional Materials. - 2013. - Vol. 20, No.3. - P. 373-377.

[6] P.E. Blochl. Projector augmented-wave method. Phys. Rev. B - 1994. - Vol. 50, No. 23. - P. 17953-17979.

[7] Patent US 8012371B2. Luminescent material. P. J. Schmidt, Th. Juestel; W. Mayr; Koninklijke Philips Electronics N.V. 2006.

[8] In-Sun Cho, Geun Kyu Choi, Jae-Sul An, Jeong-Ryeol Kim, Kug Sun Hong. Sintering, microstructure and microwave dielectric properties of rare earth orthophosphates, $\mathrm{RePO}_{4}(\mathrm{Re}=\mathrm{La}, \mathrm{Ce}, \mathrm{Nd}, \mathrm{Sm}, \mathrm{Tb}, \mathrm{Dy}, \mathrm{Y}, \mathrm{Yb})$. Materials Research Bull. - 2009. - Vol. 44. - P. 173-178

[9] P. Savchyn, I. Karbovnyk, V. Vistovskyy, A. Voloshinovskii, V. Pankratov, M. Cestelli Guidi, Mirri, O. Myahkota, A. Riabtseva, N. Mitina, A. Zaichenko, and A. I. Popov. Vibrational properties of $\mathrm{LaPO}_{4}$ nanoparticles in mid- and farinfrared domain. Journal of Applied Physics. - 2012. - V.112. - P.124309.

[10] R. D. Shannon, R. C. Shannon, O. Medenbach, R. X. Fischer. Refractive Index and Dispersion of Fluorides and Oxides. J. Phys. Chem. Ref. Data. - 2002. - Vol. 31. - P 931-970.

[11] Wide-gap luminescent materials. Theory and applications. / Edited by S. R. Rotman. - Kluwer Academic Publishers, Norwell, 1997. 
- Distribution of secondary electrons thermalization length is calculated for $\mathrm{LaPO}_{4}$ and $\mathrm{LuPO}_{4}$

- Dependence of X-ray excited luminescence intensity on the nanoparticle size was simulated

- Correlation of experimental and simulated dependencies was found

- The search criteria for scintillating nanoparticles is proposed 